\title{
MAXIMAL FUNCTIONS: A PROOF OF A CONJECTURE OF A. ZYGMUND
}

\author{
BY ANTONIO CÓRDOBA
}

In $\mathbf{R}^{n}$ let us consider the family $\boldsymbol{B}_{n}$ of parallelepipeds with sides parallel to the coordinate axes. We may ask for conditions upon the locally integrable function $f$ in order that

[*]

$$
\lim _{\substack{x \in R \in B_{n} \\ \operatorname{diam}(R) \stackrel{n}{\rightarrow} 0}} \frac{1}{\mu\{R\}} \int_{R} f(y) d \mu(y)=f(x)
$$

a.e. $x$., where $\mu=$ Lebesgue measure in $\mathbf{R}^{n}$.

In 1935 B. Jessen, J. Marcinkiewicz and A. Zygmund [1] showed that [*] holds so long as $f \in L\left(1+\left(\log ^{+} L\right)^{n-1}\right)\left(\mathbf{R}^{n}\right)$ locally. Furthermore this result is the best possible in the following sense: if $\psi(t)$ is an Orlicz's space defining function such that $\psi(t)=o\left(t(\log t)^{n-1}\right), t \rightarrow \infty$, then statement [*] is false for a typical $L_{\psi}$-function (typical in the sense of Baire's category). Of course the case $n=1$ was known before as Lebesgue's Differentiation theorem.

The following natural problem was proposed by A. Zygmund: given a positive function $\Phi$ on $\mathbf{R}^{2}$, monotonic on each variable separately, let us consider the differentiation basis $B_{\Phi}$ in $\mathbf{R}^{3}$ defined by the two parameters family of parallelepipeds whose sides are parallel to the rectangular coordinate axis and whose dimensions are given by $s \times t \times \Phi(s, t), s, t$ positive real numbers. For which locally integrable functions $f$ is statement [*] true with respect to the family $B_{\Phi}$ ?

In general the differentiation properties of $\boldsymbol{B}_{\Phi}$ must be, at least, not worse than $B_{3}$, the basis of all parallelepipeds in $\mathbf{R}^{3}$ whose sides have the direction of the coordinate axes, and, of course, not better than $\boldsymbol{B}_{2}$. A. Zygmund conjectured after his 1935 paper that $B_{\Phi}$ behaves like $B_{2}$. This conjecture is now a theorem with applications to a.e. convergence of Poisson Kernels associated to certain symmetric spaces.

THEOREM. (a) $B_{\Phi}$ differentiates integrals of functions which are locally in $L\left(1+\log ^{+} L\right)\left(\mathbf{R}^{3}\right)$, that is

Received by the editors August 29, 1978.

AMS (MOS) subject classifications (1970). Primary 42A68, 42A92; Secondary 42A18. 


$$
\lim _{\substack{R \Rightarrow x \\ R \in B_{\Phi}}} \frac{1}{\mu\{R\}} \int_{R} f(y) d \mu(y)=f(x), \text { a.e. } x
$$

so long as $f$ is locally in $L\left(1+\log ^{+} L\right)\left(\mathbf{R}^{3}\right)$, where $\mu$ denotes Lebesgue measure in $\mathbf{R}^{3}$.

(b) The associated maximal function

$$
M_{\Phi} f(x)=\operatorname{Sup}_{\substack{x \in R \\ R \in B_{\Phi}}} \frac{1}{\mu\{R\}} \int_{R}|f(y)| d \mu(y)
$$

satisfies the inequality

$$
\mu\left\{M_{\Phi} f(x)>\alpha>0\right\} \leqslant C \int_{\mathbf{R}^{3}} \frac{|f(x)|}{\alpha}\left\{1+\log +\frac{|f(x)|}{\alpha}\right\} d \mu(x)
$$

for some universal constant $C<\infty$.

The proof is based on the following geometric argument:

Covering Lemma. Let $B$ be a family of dyadic parallelepipeds in $\mathbf{R}^{\mathbf{3}}$ satisfying the following monotonicity property: if $R_{1}, R_{2} \in B$ and the horizontal dimensions of $R_{1}$ are both strictly smaller than the corresponding dimensions of $\mathbf{R}^{2}$, then the vertical dimension of $R_{1}$ must be not bigger than the vertical dimension of $R_{\mathbf{2}}$.

Then the family $B$ satisfies the exponential type covering property, that is: Given $\left\{R_{\alpha}\right\} \subset B$ one can select a subfamily $\left\{R_{f}\right\} \subset\left\{R_{\alpha}\right\}$ such that,

(i) $\mu\left\{\bigcup R_{\alpha}\right\} \leqslant C \mu\left\{\bigcup R_{j}\right\}$,

(ii) $\int \bigcup_{R_{j}} \exp \left(\Sigma \chi_{R_{j}}(x)\right) d \mu(x) \leqslant C \mu\left\{\bigcup R_{j}\right\}$

for some universal constant $C<\infty$.

Application. Consider

$$
\mathbf{R}^{3}=\left\{X=\left(\begin{array}{ll}
x_{1} & x_{3} \\
x_{3} & x_{2}
\end{array}\right), \text { real, symmetric, } 2 \times 2 \text {-matrices }\right\},
$$

and the cone $\Gamma=\left\{X \in \mathbf{R}^{3}\right.$, positive definite $\}$. Then $T_{\Gamma}=$ tube over $\Gamma=$ Siegel's upper half-space $=\{X+i Y, Y$ positive definite $\}$.

For each integrable function $f$ in $\mathbf{R}^{\mathbf{3}}$ we have the "Poisson integral",

$$
u(X+i Y)=P_{Y} * f(x), \quad Y \in \Gamma
$$

where

$$
P_{Y}(X)=C[\operatorname{det} Y]^{3 / 2} /|\operatorname{det}(X+i Y)|^{3}
$$

and we may ask the following question: for which functions $f$ is it true that $u(X+i Y) \rightarrow f(X)$, a.e. $X$, where $Y \rightarrow 0$ ? 
It is a well-known fact that if $Y=c I=\left(\begin{array}{cc}c & 0 \\ 0 & c\end{array}\right) \rightarrow 0$, then $u(X+i Y) \rightarrow$ $f(X)$, a.e. $X$ for integrable functions $f$. On the other hand if $Y \rightarrow 0$ without any restriction than a.e. convergence fails for every class $L^{p}\left(\mathbf{R}^{3}\right), 1 \leqslant p \leqslant \infty$.

Here we can settle the case

$$
Y=\left(\begin{array}{ll}
y_{1} & 0 \\
0 & y_{2}
\end{array}\right) \rightarrow 0
$$

because an easy computation shows that

$$
M f(X)=\operatorname{Sup}_{Y}|u(X+i Y)|
$$

where

$$
Y=\left(\begin{array}{ll}
y_{1} & 0 \\
0 & y_{2}
\end{array}\right)
$$

is majorized, in a suitable sense, by $M_{\Phi} f$ with $\Phi(s, t)=(s \cdot t)^{1 / 2}$. Therefore we have convergence for $L\left(1+\log ^{+} L\right)\left(\mathbf{R}^{3}\right)$ and, since $M f \geqslant C M_{\Phi} f$ is also true for some $c>0, L\left(1+\log ^{+} L\right)\left(\mathbf{R}^{3}\right)$ is the best class for which almost everywhere convergence holds.

\section{REFERENCES}

1. B. Jessen, J. Marcinkiewicz and A. Zygmund, Note on the differentiability of multiple integrals, Fund. Math. 25 (1935), 217-234.

2. A. Córdoba, $s \times t \times \Phi(s, t)$, Institut Mittag-Leffler Report No. 9, 1978.

3. E. M. Stein and N. J. Weiss, On the convergence of Poisson integrals, Trans. Amer. Math. Soc. 140 (1969), 34-54.

DEPARTMENT OF MATHEMATICS, PRINCETON UNIVERSITY, PRINCETON, NEW JERSEY 08540 SPAIN

FACULTAD DE MATHEMATICAS, UNIVERSIDAD COMPLUTENSE, MADRID, 